Original Paper http://ajol.info/index.php/ijbcs http://indexmedicus.afro.who.int

\title{
Analyse comparative entre la distribution des fréquences de conductivité électrique et les faciès géochimiques des eaux des aquifères de socle (Côte d'Ivoire)
}

\author{
Amani Michel KOUASSI ${ }^{1 *}$, Kouassi Ernest AHOUSSI ${ }^{2}$, Koffi Eugène KOUAKOU ${ }^{1}$, \\ Amos MAMADOU $^{3}$ et Jean BIEMI ${ }^{2}$ \\ ${ }^{1}$ Institut National Polytechnique Félix Houphouët-Boigny (INP-HB) ; Département des Sciences de la Terre et \\ des Ressources Minières (STeRMi) ; Laboratoire du Génie Civil, des Géosciences et des Sciences \\ Géographiques ; BP 1093 Yamoussoukro, Tél. (+225) 306467 15, Côte d’Ivoire. \\ ${ }^{2}$ Université Félix Houphouët-Boigny d'Abidjan-Cocody, Unité de Formation et de Recherche des Sciences de \\ la Terre et des Ressources Minières (UFR-STRM) ; Laboratoire des Sciences et Techniques de l'Eau et de \\ l'Environnement (LSTEE) ; 22 BP 582 Abidjan 22 ; Tel : (+225) 224838 03, Côte d'Ivoire. \\ ${ }^{3}$ Institut National Polytechnique Félix Houphouët-Boigny (INP-HB) de Yamoussoukro ; Ecole Supérieure des \\ Mines et de Géologie (ESMG) ; BP 1093 Yamoussoukro; Tél. : (+225) 3064 36 07, Côte d'Ivoire. \\ "Auteur Correspondant ; E-mail : michel.a_kouassi@yahoo.fr
}

\section{RÉSUMÉ}

La conductivité électrique est un indicateur de la minéralisation totale des eaux et son étude permet de faire un suivi hydrochimique des systèmes hydrologiques. L'objectif de ce travail est d'analyser l'apport de l'étude de la distribution des fréquences de conductivité électrique à la caractérisation géochimique des eaux des aquifères fissurés de socle cristallin et cristallophyllien. Les zones tests utilisées dans le cadre de ce travail appartiennent au socle ivoirien. La méthodologie adoptée est basée d'une part sur l'étude des courbes de fréquence de conductivité électrique. D'autre part, les faciès chimiques des eaux souterraines ont été déterminés à partir du diagramme de Piper. L'analyse de la distribution des fréquences de conductivité électrique a fait ressortir diverses familles d'eau. La projection des échantillons d'eau sur le diagramme de Piper a permis de mettre en évidence plusieurs faciès des eaux souterraines. La comparaison des différents résultats met en évidence une concordance au niveau de quatre échantillons (Bondoukou, Tiassalé, ex-N'ziComoé et Tiébissou-Didievi) et une non concordance au niveau de deux échantillons (Guiglo-Duekoué et Agboville). On peut conclure que l'analyse des fréquences de conductivité électrique permet en général de confirmer les différents faciès hydrogéochimiques.

(C) 2016 International Formulae Group. All rights reserved.

Mots clés: Hydrogéochimie, conductivité électrique, diagramme de Piper, socle cristallin et cristallophyllien, Côte d'Ivoire.

\section{Comparative analysis of electrical conductivity frequencies distribution and geochemical facies of hard rock aquifers waters (Ivory Coast)}

\begin{abstract}
Electrical conductivity is a measure of the total mineralization of the water and its study allows for a hydrochemical monitoring of water systems. The objective of this work was to analyze the contribution of the
\end{abstract}


study of electrical conductivity frequencies distribution to the geochemical characterization of the hard rock aquifers waters. The test zones used in the context of this work belong to the Ivorian hard rock aquifers. The methodology is based firstly on the study of the frequency curves of the electrical conductivity. On the other hand, chemical facies of groundwater have been determined from the Piper diagram. The analysis of the distribution of the electrical conductivity of frequencies has highlighted various water families. The projection of water samples on the Piper diagram helped highlight several facies of groundwater. Comparing the results of different methodological approaches reveals a match in four samples (Bondoukou, Tiassalé, N'zi-Comoé and Tiébissou-Didievi) and a mismatch in two samples (Guiglo-Duékoué and Agboville). Given concordances results in most cases, we can say that the analysis of the electrical conductivity of frequencies generally allows confirming the different hydrogeochemical facies in the hard rock aquifers.

(C) 2016 International Formulae Group. All rights reserved.

Keywords: Hydrogeochemistry, electrical conductivity, Piper diagram, hard rock aquifers, Ivory Coast.

\section{INTRODUCTION}

La conductivité électrique est un indicateur de la minéralisation totale des eaux. Ainsi, l'étude de la conductivité électrique permet de faire un suivi hydrochimique des systèmes hydrologiques (Fournier, 2006 ; Kouassi et al., 2012a). En effet, le suivi d'un cycle hydrologique permet d'étudier les variations saisonnières du chimisme des eaux. En relation directe avec les évènements hydrologiques (pluie, neige), les variations du chimisme des eaux traduisent l'hétérogénéité du fonctionnement de l'aquifère, d'un point de vue spatial (zone noyée et zone d'infiltration) et d'un point de vue temporel (étiage, hautes eaux). De par sa capacité à synthétiser les données volumineuses, l'étude de la fréquence des données est réalisée dans plusieurs domaines (hydrologie, hydrogéologie, etc.). C'est ainsi que Massei (2001) a introduit le suivi en continue à haute fréquence de la hauteur d'eau et de turbidité des systèmes afin de comparer les variations relatives des marqueurs de l'hydrodynamisme, des éléments dissous et particulaires. Ainsi, à partir de ces variations couplées aux informations obtenues par l'analyse microgranulométrique de la fraction particulaire et aux observations au microscope électronique à balayage (MEB), l'auteur a identifié la nature et l'origine de la turbidité. Les courbes normalisées de fréquence de conductivité électrique, de turbidité et de débit ont été utilisées par Fournier (2006) pour mettre en évidence les modalités de transport à l'échelle d'une crue.
Aussi, l'analyse dans l'espace des fréquences de conductivité électrique permet-elle de déterminer les familles d'eau qui définissent les faciès chimiques des eaux souterraines (Fournier, 2006). En effet, il existerait une adéquation entre la famille d'eau identifiée à partir de l'analyse de la distribution des fréquences de conductivité électrique et les faciès chimiques des eaux déterminés à partir des diagrammes hydrochimiques (diagramme de Piper, diagramme de Shöeller-Berkaloff, diagramme rayonnant, etc.). L'hypothèse de travail a consisté à supposer qu'il existe une concordance entre les familles d'eau mises en évidence par l'étude de la distribution des fréquences de conductivité électrique et les faciès chimiques des eaux souterraines identifiés à partir des digrammes de minéralisation.

L'objectif général de cette étude vise à l'analyse hydrogéochimique des eaux des formations cristallines et cristallophylliennes à partir de l'étude de la conductivité électrique. Cela permet de dégager trois objectifs spécifiques qui sont :

i) identifier les différents modes de distribution des fréquences de la conductivité électrique des eaux souterraines ;

ii) déterminer les faciès chimiques des eaux souterraines ;

iii) analyser l'adéquation entre les modes de distribution des fréquences et les faciès chimiques des eaux souterraines.

La méthodologie adoptée a consisté d'une part à analyser la distribution des fréquences de la conductivité électrique des 
eaux souterraines et d'autre part, déterminer les faciès chimiques des eaux à partir du diagramme de minéralisation de Piper.

\section{MATÉRIEL ET MÉTHODES}

\section{Présentation de la zone d'étude}

La Côte d'Ivoire fait partie des pays du Golfe de Guinée. Elle s'étend sur une superficie de $322462 \mathrm{Km}^{2}$, environ $1 \% \mathrm{du}$ continent africain. Ses frontières dessinent approximativement un carré s'inscrivant entre les coordonnées de $2^{\circ} 30^{\prime}$ et $8^{\circ} 30^{\prime}$ de longitude Ouest, $4^{\circ} 30^{\prime}$ et $10^{\circ} 30^{\prime}$ de latitude Nord avec, au Sud, une façade littorale de $550 \mathrm{~km}$. Elle est limitée au Nord par le Mali et le Burkina Faso, à l'Est par le Ghana, à l'Ouest par la Guinée et le Libéria et au Sud par l'océan atlantique. La Figure 1 montre la situation de la Côte d'Ivoire en Afrique de l'Ouest et les zones de prélèvement des échantillons d'eaux souterraines à partir de forages.

Deux grandes zones climatiques se côtoient: le climat équatorial et le climat tropical. Le climat subéquatorial dans le Sud est caractérisé par des précipitations abondantes, qui atteignent en moyenne 1 $800 \mathrm{~mm}$. Le climat tropical humide de savane couvre le Centre du pays. Les précipitations annuelles moyennes sont proches de $1200 \mathrm{~mm}$. Le climat tropical atténué concerne principalement la partie Nord du pays et les précipitations moyennes annuelles enregistrées sont généralement inférieures à $1000 \mathrm{~mm}$. Le régime climatique régnant au niveau de l'Ouest de la Côte d'Ivoire est un climat de montagne avec des hauteurs pluviométriques annuelles dépassant parfois les $2000 \mathrm{~mm}$. Deux grands types de paysages végétaux se partagent le territoire ivoirien : un paysage forestier et un paysage de savane. Le premier correspond à la moitié Sud du pays et appartient au domaine guinéen. Le second occupe la moitié Nord de la Côte d'Ivoire et se rattache au domaine soudanais (Brou, 2005). Le socle ivoirien est composé des domaines archéen, paléoprotérozoïque et Sassandra-Cavally (SASCA). Le domaine archéen a été affecté par des déformations tectoniques très importantes attribuées à l'orogenèse léonienne (3500-2900 Ma) et à l'orogenèse libérienne (2900-2500 Ma). Il est caractérisé par les reliefs les plus accidentés de la Côte d'Ivoire. Le domaine archéen est dominé par des granites, des migmatites, des granito-gneiss, des amphibolo-pyroxénites, des charnockites. Le domaine paléoprotérozoïque est subdivisé en deux grands sous-ensembles (ensemble des granitoïdes et formations volcanosédimentaires). Il a été structuré au cours du mégacycle éburnéen. $\mathrm{Ce}$ domaine est constitué d'un socle granito-gneissique dominé par les granites et de formations volcano-sédimentaires (métasédiments et roches vertes). Les métasédiments sont constitués en majeure partie de grès et de schistes. Les roches vertes sont constituées de métabasalte, métaandésite, métadolérite, métagabbro, amphibolite. Il existe un autre domaine où coexistent les formations géologiques des deux grands domaines (domaine archéen et domaine paléoprotérozoïque): c'est le domaine Sassandra-Cavally (SASCA). Ce domaine est situé dans le Sud-Ouest de la Côte d'Ivoire depuis Toulepleu, Soubré, Sassandra, Taï jusqu'au fleuve Cavally et est drainé par les fleuves Sassandra et Cavally. Sa particularité est qu'on y trouve à la fois des roches issues de l'orogenèse libérienne et des orogenèses les plus récentes (Burkinienne et Eburnéenne). Les formations rencontrées dans le domaine Sassandra-Cavally (SASCA) sont les schistes, les micaschistes, les arkoses, les quartzites, les tufs métamorphiques, les metarhyolites, les amphibolites, les metagabbros, les conglomérats, des tufs métamorphiques, les migmatites, les quartzites, les gneiss et les leptynites.

En Côte d'Ivoire, les réservoirs d'eaux souterraines de socle se rencontrent dans les horizons d'altérites ainsi que dans les séries volcano-sédimentaires et granitomigmatitiques. Les réservoirs d'altérites varient selon la nature du socle. L'eau circule dans les roches cristallines à travers les réseaux de fractures à fonction drainante, généralement bien alimentés et capables de 
fournir des débits assez importants quand ils sont bien captés par les forages. En effet, le socle cristallin et cristallophyllien de Côte d'Ivoire a été affecté par les phénomènes tectoniques en y laissant d'importants réseaux de fissures plus ou moins connectés qui sont à l'origine de la formation des nappes souterraines (Lasm et al., 2004; Jourda et al., 2006a; Jourda et al., 2006b ; Lasm et al., 2008; Youanta et al., 2008; Koïta et al., 2010; N'go et al., 2010; Sorokoby et al., 2010). A l'heure actuelle, les forages captant les fractures sont les plus sûrs et les moins influençables par les fluctuations saisonnières.

\section{Collecte de données}

L'étude est basée sur six échantillons composés de plusieurs forages d'eau issus de différentes régions géologiques de la Côte d'Ivoire. Les données concernent les résultats d'analyses des paramètres physicochimiques réalisées sur ces forages. Le seul paramètre physique utilisé concerne la conductivité électrique. Les paramètres chimiques sélectionnés sont d'une part les cations majeurs (le calcium, le magnésium, le sodium, le potassium) et d'autre part, les anions majeurs (le bicarbonate, le chlore, le nitrate, le sulfate). La conductivité électrique a été mesurée in situ. En ce qui concerne les zones de l'exrégion du N'zi-Comoé, Guiglo-Duekoué, Tiébissou-Didiévi, Bondoukou et Tiassalé, les échantillons d'eau ont été analysés au Laboratoire d'Analyse d'Abengourou (LPA) et ceux d'Agboville ont été analysés au Laboratoire National d'Essai de Qualité, de Métrologie et d'Analyse (LANEMA). Les données de cette étude sont issues de plusieurs projets et programmes d'hydraulique villageoise réalisés en Côte d'Ivoire. Les données de conductivité électrique utilisées ont été corrigées de l'effet de la température.

\section{Méthodes de traitement des données Distribution des fréquences de conductivité électrique des eaux souterraines}

L'étude de la fréquence de conductivité électrique est une méthode particulière utilisée pour le traitement des données hydrochimiques (Fournier, 2006). Elle permet de mettre en évidence l'hétérogénéité de la composition chimique des eaux souterraines. Ces variations de la fréquence de la conductivité sont l'expression à la fois de l'hétérogénéité spatiale de l'aquifère, c'est-àdire de sa structure, et de processus particuliers. Elles traduisent le fait qu'il existe dans l'aquifère des eaux possédant des histoires différentes (temps de séjour, conditions d'écoulement, stockage près de la surface, etc.) et que ces eaux sont plus ou bien mélangées. Une forme unimodale de la distribution des fréquences de la conductivité de l'eau, obtenue à partir d'un suivi dans le temps, indique l'absence d'organisation de drainage au sein de l'aquifère (cas des aquifères poreux), alors que des courbes plurimodales indiquent une organisation du réseau système de drainage (cas des aquifères karstiques). En outre, il a été démontré qu'il existe une relation entre la forme de la distribution de fréquences de la conductivité électrique de l'eau d'une source et le développement du réseau de drainage fonctionnel de l'aquifère (Fournier, 2006).

L'étude de la distribution obéit à la procédure suivante :

i) la détermination du nombre de classe ;

ii) l'évaluation de l'intervalle de classe et la définition des classes;

iii) la définition des effectifs puis des fréquences par classe ;

iv) la représentation des fréquences de conductivité électrique.

Le nombre de classe a été déterminé à partir des méthodes de Sturge et de Yule (Kouassi et al., 2014):

- la règle de Sturge:

- $\quad N=1+(3,3 \log n)$

- la règle de Yule:

- $N=2,5 n^{1 / 4}$

L'intervalle de classe (I) a été défini comme suit (équation 3):

$I=\frac{\left(C E_{\max }-C E_{\min }\right)}{N}$

-I : intervalle de classe ; 
- $\mathrm{CE}_{\max }$ : valeur maximale de conductivité électrique de la série considérée ;

- $\mathrm{CE}_{\min }$ : valeur minimale de conductivité électrique de la série considérée ;

$-\mathrm{N}$ : nombre de classe.

A partir de la borne inférieure, on obtient les limites de classes ou bornes de classes par addition successive de l'intervalle de classe. En règle générale, on tente de faire coïncider l'indice de classe ou valeur centrale de la classe avec un nombre entier.

Les courbes de fréquence de conductivité électrique ont été effectuées à partir des fréquences de conductivité électrique calculées à partir de l'effectif par classe (Ni) et de l'effectif total de l'échantillon (N) (équation 4):

$F_{i}=100 \times \frac{N_{i}}{N}$

Les différents traitements ont été réalisés à l'aide du logiciel Microsoft Excel.

Détermination des faciès chimiques des eaux souterraines

L'analyse hydrogéochimique a été réalisée à l'aide du digramme de Piper. Ce diagramme a été utilisé pour caractériser les faciès géochimiques des eaux de la zone d'Agboville et de Tiébissou-Didievi. En effet, les échantillons des autres zones tests ont déjà fait l'objet d'étude par plusieurs auteurs à partir du diagramme de Piper. Il s'agit des échantillons de Tiassalé (Oga et al., 2009), ex-N'zi-Comoé (Kouassi et al., 2010), Guiglo-Duekoué (Kouassi et al., 2012b) et Bondoukou (Ahoussi et al., 2012a). Le digramme de Piper est très fréquemment utilisé et donne de très bons résultats (Yermani et al., 2003 ; Mohammad et Hamed, 2004 ; Tabouche et Achour, 2004 ; Alayat et Lamouroux, 2007 ; Kouzana et al., 2007 ; Kouassi et al., 2010; Yao et al., 2010 ; Ahoussi et al., 2011 ; Ahoussi et al., 2012b). Les différents traitements ont été possibles grâce au logiciel DIAGRAMME ${ }^{\mathrm{TM}}$.

\section{RÉSULTATS}

Résultats d'analyse des fréquences de la conductivité électrique

Les courbes de fréquences de conductivité électrique obtenues ont été classées en trois principaux groupes: les courbes unimodales, les courbes bimodales et les courbes plurimodales.

Des courbes unimodales ont été obtenues au niveau des échantillons de l'exrégion du N'zi-Comoé et d'Agboville (Figures 2). La courbe de fréquence de l'ex-région du N'zi-Comoé est caractérisée par une classe modale allant de 249 à $392 \mu \mathrm{S} / \mathrm{cm}$, avec une fréquence de 32,64\%. Quant à la courbe de fréquence de la zone d'Agboville, elle est caractérisée par une classe modale allant de 158 à $237 \mu \mathrm{S} / \mathrm{cm}$ avec une fréquence de $32,43 \%$. Ces courbes traduisent la présence d'une seule famille d'eau prépondérante dans ces zones.

Les conductivités électriques de la zone de Tiébissou-Didiévi sont gouvernées par deux valeurs essentielles (Figure 3). La première classe modale est $104-203 \mu \mathrm{S} / \mathrm{cm}$ avec une fréquence de $25,93 \%$ et la deuxième classe modale est $401-500 \mu \mathrm{S} / \mathrm{cm}$, caractérisée par une fréquence de $20 \%$. La région de Bondoukou présente une première classe modale axée autour de l'intervalle 134-244 $\mu \mathrm{S} / \mathrm{cm}$ avec une fréquence maximale de $44,68 \%$ et une deuxième classe modale qui est de $464-574 \mu \mathrm{S} / \mathrm{cm}$ avec une fréquence maximale de $12,77 \%$ (Figure 3). Les conductivités électriques de la zone de Tiassalé sont marquées par les valeurs axées autour des classes modales 348-583 $\mu \mathrm{S} / \mathrm{cm}$ $(35,18 \%)$ et $1993-2228 \mu \mathrm{S} / \mathrm{cm}(6 \%)$ (Figure 3). La structure de ces courbes indique la présence de deux familles d'eau dans ces aquifères. Un autre constat est que les pics que présentent ces courbes sont inégaux. Ainsi, quel que soit le cas, un des pics présente une fréquence élevée tandis que le deuxième présente une fréquence basse. Cela traduit une inégale répartition des familles d'eau dans les aquifères considérés. Il y aurait alors la présence d'une famille prédominante par rapport à l'autre. 
La région de Guiglo-Duekoué est marquée par une succession de quatre (4) pics (Figure 4). Ce qui traduit la présence de quatre familles d'eau essentielles. La première classe est axée autour de la classe 106-130 $\mu \mathrm{S} / \mathrm{cm}$ avec une fréquence de $25 \%$. La deuxième classe est située autour de 154-202 $\mu \mathrm{S} / \mathrm{cm}$ avec une fréquence de $10,42 \%$. La troisième classe est caractérisée par l'intervalle 226-250 $\mu \mathrm{S} / \mathrm{cm}$ avec une fréquence de $14,58 \%$ et enfin une quatrième classe située autour de 274-298 $\mu \mathrm{S} / \mathrm{cm}$ avec une fréquence de $16,67 \%$. La répartition de ces quatre pics se fait de telle sorte que le premier est beaucoup plus significatif ensuite vient les trois autres qui évoluent de façon croissante. Ainsi, la famille d'eaux axée autour de la conductivité de 106$130 \mu \mathrm{S} / \mathrm{cm}$ est prépondérante tandis que les autres familles demeurent mineures.

\section{Résultats de l'étude des faciès chimiques des eaux souterraines}

La projection des échantillons d'eau sur le diagramme de Piper a permis de mettre en évidence les différentes familles d'eau présentes dans les différentes régions de Tiébissou-Didievi et d'Agbovile. Au niveau de la région de Tiébissou-Didievi, il est mis en évidence trois familles d'eau qui sont les eaux bicarbonatées, calciques et magnésiennes $(65,38 \%)$, les eaux bicarbonatées sodipotassiques $(30,77 \%)$ et les eaux chlorurées et sulfatées, calciques et magnésiennes $(3,85 \%)$ (Figure 5). Les eaux bicarbonatées, calciques et magnésiennes et les eaux bicarbonatées sodi-potassiques constituent les faciès prédominants. Il est constaté la prédominance du faciès bicarbonaté $(96,15 \%)$ sur le faciès chloruré $(3,85 \%)$. La représentation des points d'eau du domaine cristallin d'Agboville met en évidence quatre familles d'eau qui sont les eaux bicarbonatées, calciques et magnésiennes $(61,90 \%)$, les eaux chlorurées, sodiques et potassiques $(21,43 \%)$, les eaux bicarbonatées, sodiques et potassiques $(9,52 \%)$ et les eaux chlorurées et sulfatées calciques et magnésiennes (7,14\%) (Figure 5). Les eaux bicarbonatées, calciques et magnésiennes et les eaux chlorurées, sodiques et potassiques constituent les faciès dominants. Il est constaté la prédominance du faciès bicarbonaté $(71,42 \%)$ sur le faciès chloruré $(28,58 \%)$.

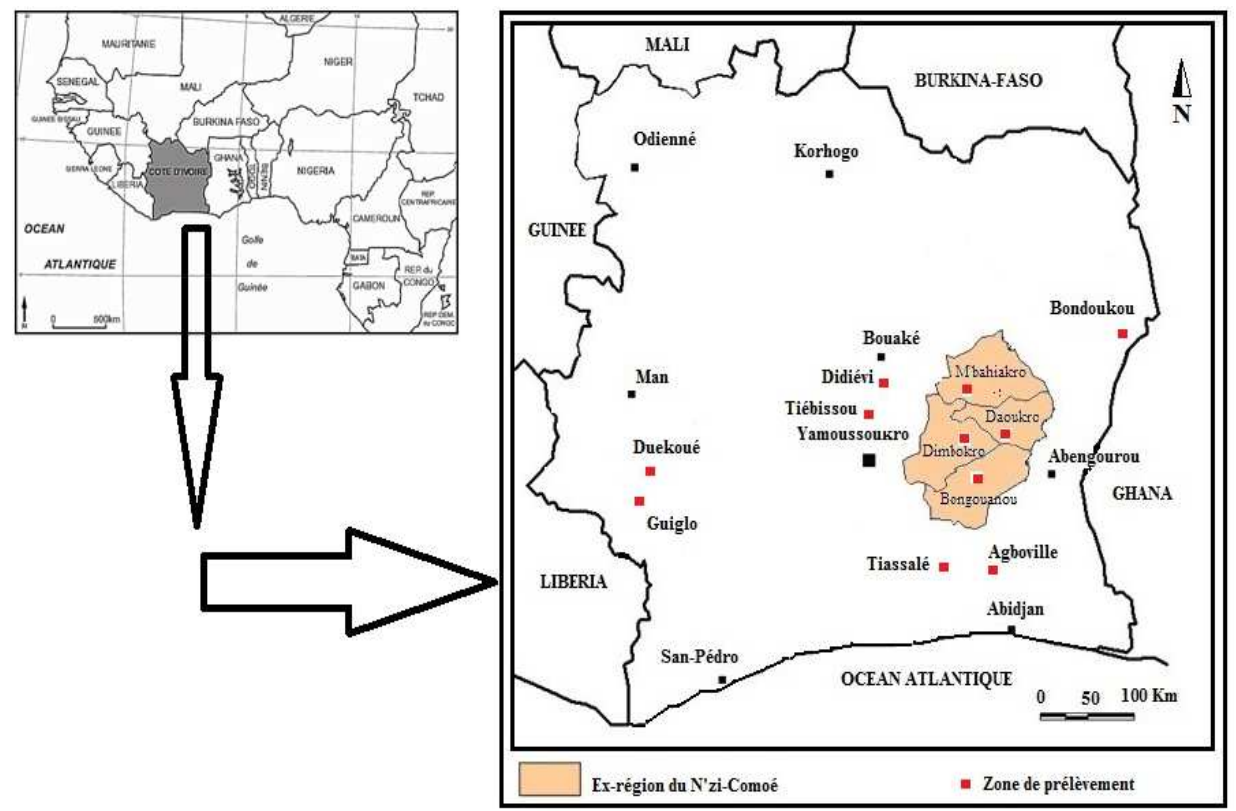

Figure 1: Zones de prélèvement des échantillons d'eaux souterraines à partir de forages. 

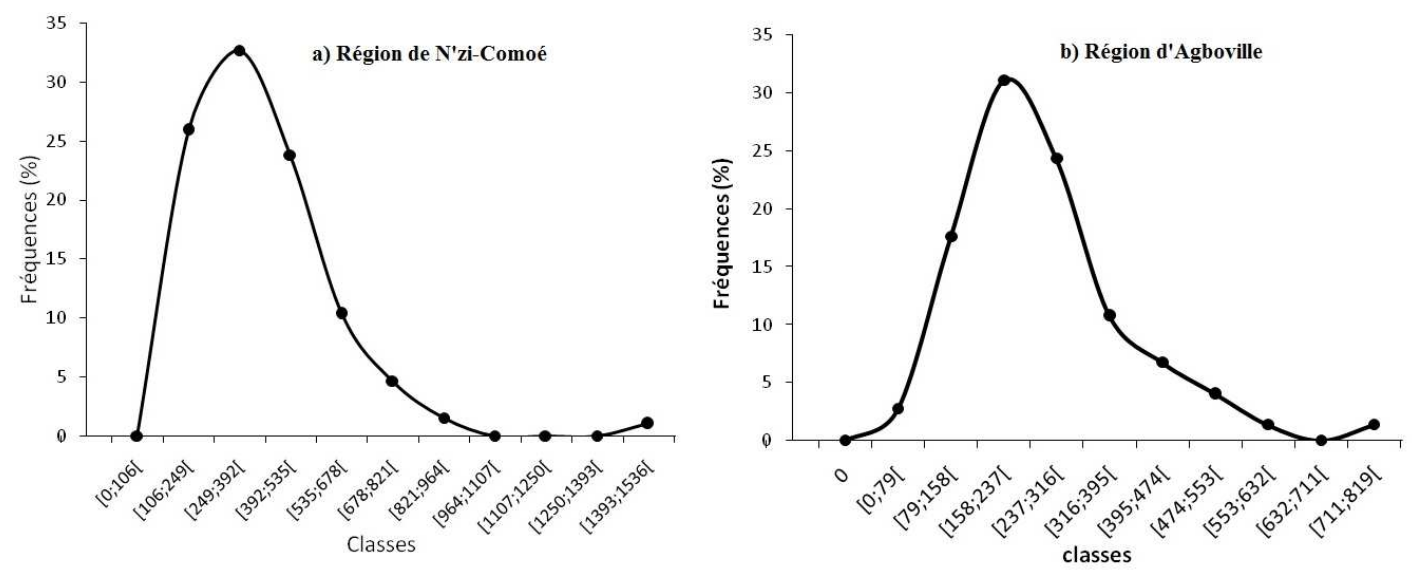

Figure 2: Fréquences de conductivité électrique dans les régions du N'zi-Comoé (a) et d'Agboville (b).
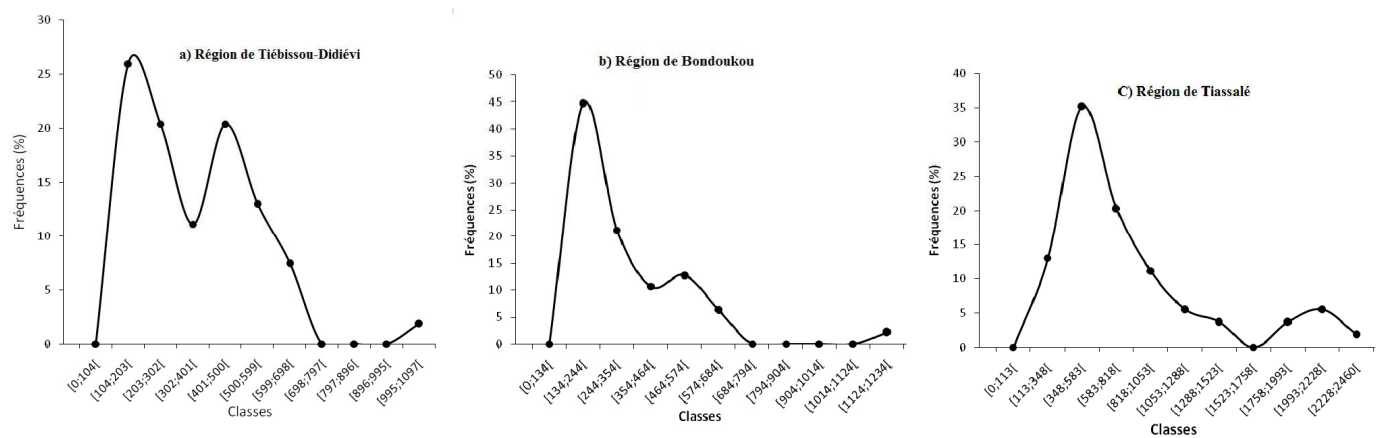

Figure 3: Fréquences de conductivité électrique dans les régions de Tiébissou-Didiévi (a), Bondoukou (b) et Tiassalé (c).

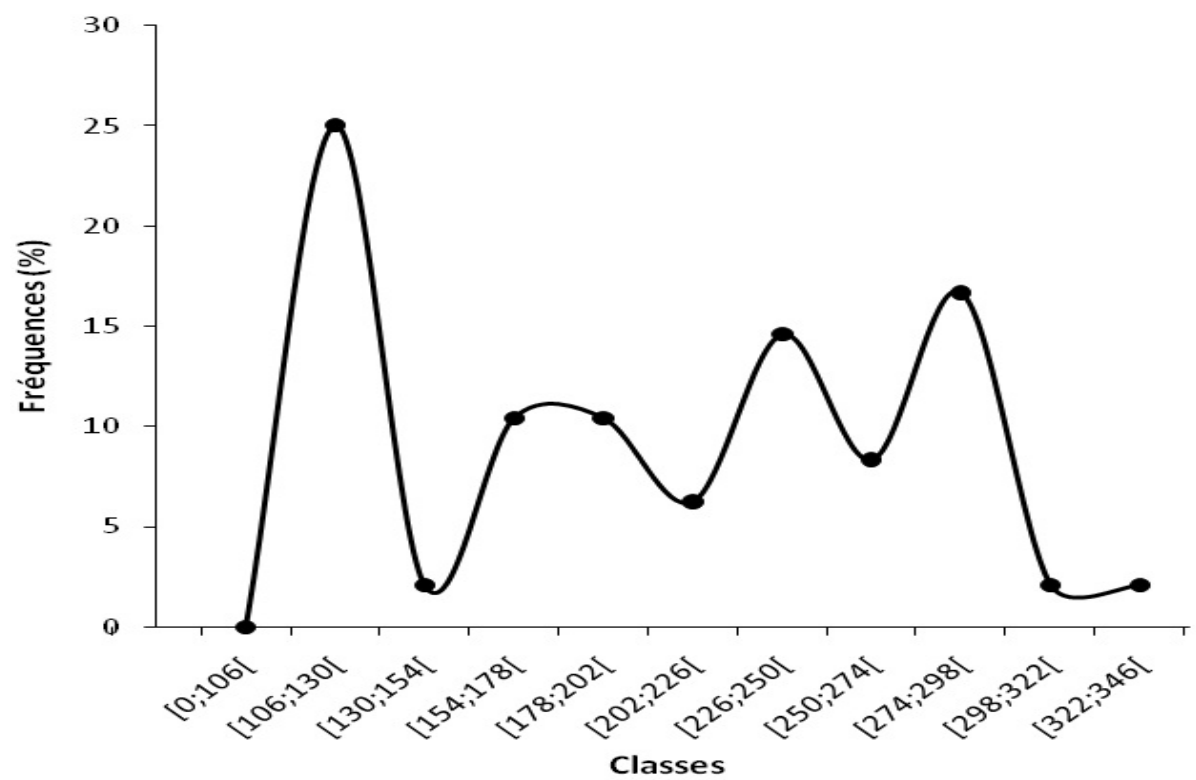

Figure 4: Fréquences de conductivité électrique au niveau de Guiglo-Duekoué. 

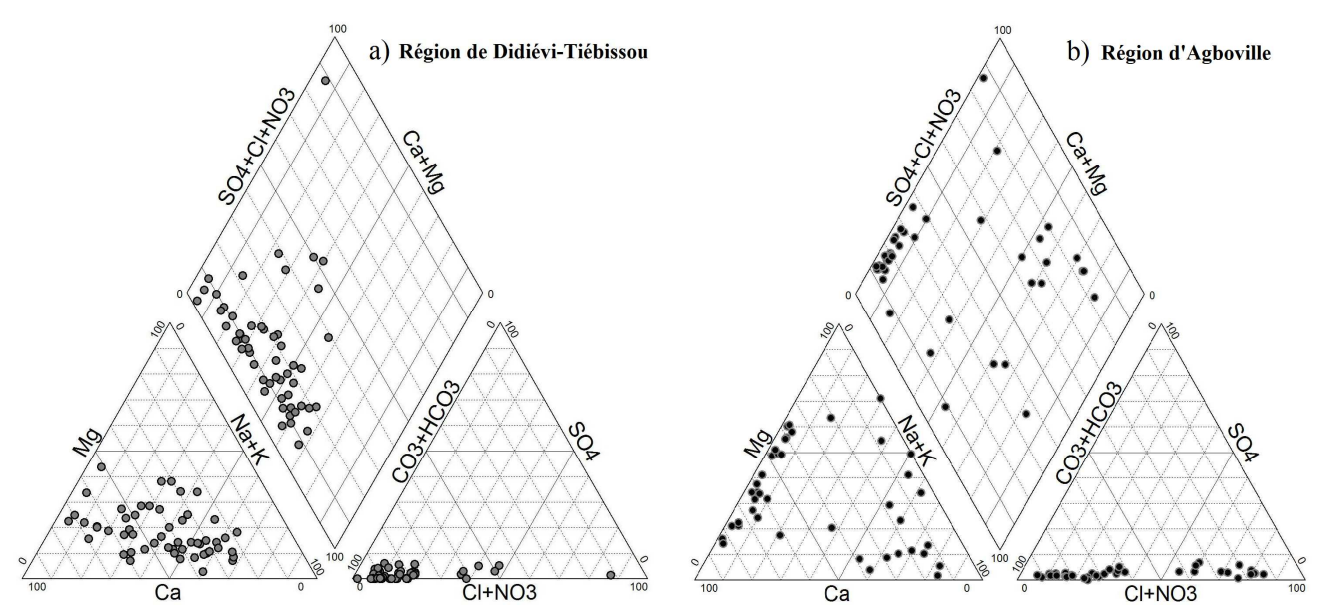

Figure 5: Diagrammes de Piper des régions de Didievi-Tiébissou et Agboville.

\section{DISCUSSION}

L'analyse de la distribution des fréquences de conductivité électrique des différentes zones tests a mis en évidence les différentes familles d'eaux présentes dans les aquifères de ces zones. Ces familles d'eaux ont été traduites par les formes des courbes de fréquences de la conductivité électrique. En fonction du nombre de pics, il a été identifié des courbes unimodales (ex-région du N'ziComoé et Agboville), bimodales (TiébissouDidiévi, Tiassalé et Bondoukou) et plurimodales (4) (Guiglo-Duekoué).

Les résultats de l'application du diagramme de minéralisation de Piper a mis en évidence au niveau de la région de Tiébissou-Didievi, trois familles d'eau dont les faciès dominants sont les eaux bicarbonatées, calciques et magnésiennes $(65,38 \%)$ suivis des eaux bicarbonatées sodipotassiques $(30,77 \%)$. Au niveau de la région d'Agboville, il a été mis en évidence quatre familles d'eau dont les plus prédominants sont les eaux bicarbonatées, calciques et magnésiennes (61,90\%). L'étude de la caractérisation des eaux souterraines dans l'ex-région du N'zi-Comoé effectuée par Kouassi et al. (2010) ont révélé deux faciès: le faciès bicarbonaté calcique et magnésien et le faciès chloruré calcique et magnésien. Ils ont donc montré que les eaux bicarbonatées calciques et magnésiennes sont les plus dominantes dans l'ex-région du N'zi-Comoé et sont présentes dans tous les départements. Quatre-vingt-dix-neuf pourcent $(99 \%)$ des points d'eau de la zone se rangent dans cette classe. Par ailleurs, les eaux chlorurées calciques et magnésiennes sont très rares dans la région et elles représentent seulement $1 \%$ des points d'eau. Les travaux de Oga et al. (2009) dans la région de Tiassalé ont mis en évidence deux faciès chimiques des eaux souterraines. Le faciès bicarbonaté, calcique et magnésien qui se rencontre dans $83 \%$ des eaux de la région et le faciès chloruré calcique et magnésien qui concerne $17 \%$ des échantillons. La classification hydrochimique des eaux dans le diagramme de Piper effectué par Ahoussi et al. (2012a) dans la région de Bondoukou ont mis en évidence deux principaux hydrofaciès. Il s'agit des eaux biacrbonatéess calciques $(93,75 \%)$ et des eaux chlorurées calciques $(6,25 \%)$. Les travaux de Kouassi et al. (2012b) dans la région de Guiglo-Duekoué ont mis en évidence deux principales familles d'eau qui sont les eaux bicarbonatées calciques et magnésiennes $(95,83 \%)$ et les eaux bicarbonatées sodipotassiques $(4,17 \%)$.

Une comparaison des différents résultats issus de l'analyse des courbes de fréquence de conductivité électrique et de l'interprétation des diagrammes de 
minéralisation de Piper a mis en évidence deux tendances.

Une concordance constatée au niveau des échantillons de Bondoukou, Tiassalé, exN'zi-Comoé et de Tiébissou-Didievi. En effet, des courbes bimodales ont été identifiées avec deux faciès hydrogéochimiques au niveau des échantillons de Bondoukou et Tiassalé. Une courbe unimodale avec un faciès prédominant ont été obtenus au niveau de l'ex-N'ziComoé. En effet, ces résultats confirment bien la présence d'une seule famille d'eau révélée par l'analyse de la fréquence des conductivités électriques vu que toutes les eaux peuvent être rangées globalement dans la famille des eaux bicarbonatées calciques et magnésiennes représentant $99 \%$ des points d'eau analysés. Une concordance est aussi constatée au niveau de Tiébissou-Didievi. En effet, une courbe bimodale a été identifiée au niveau de Tiébissou-Didievi alors que le diagramme de minéralisation a mis en évidence trois familles d'eau dont deux faciès dominants. On pourrait donc assimiler les deux familles d'eau déterminées par les courbes de conductivité électrique aux deux faciès prédominants révélés par le diagramme de Piper. Cette adéquation entre les résultats des différentes approches méthodologiques confirme l'hypothèse de base ;

Deux cas de non concordance ont été constatés au niveau de l'échantillon de Guiglo-Duekoué et d'Agboville. En effet, une courbe plurimodale (4) a été obtenue au niveau de Guiglo-Duekoué contre deux faciès chimiques. Ainsi, les eaux bicarbonatées calciques et magnésiennes représentent la première famille axée dans la classe 0-154 $\mu \mathrm{S} / \mathrm{cm}$ avec $25 \%$ de fréquence indiquée par la fréquence des conductivités électriques. La famille des eaux bicarbonatées sodipotassique pourrait regrouper les trois dernières familles distinguées lors de l'analyse des fréquences des conductivités électriques. L'évolution en dents de scie observée dans cette famille est due à une grande variation de la conductivité électrique au sein des eaux bicarbonatées sodi-potassiques. Cette divergence des résultats pourrait s'expliquer par la mauvaise balance observée au sein de la série (Kouassi et al., 2013). Au niveau d'Agboville, une courbe unimodale de conductivité électrique contre quatre faciès hydrogéochimiques dont deux faciès dominants ont été obtenus. Pourtant, l'échantillon d'Agboville a présenté une bonne balance ionique (Kouassi et al., 2013). Ces résultats ne corroborent pas l'hypothèse de base et pourraient s'expliquer par sa situation géographique. En effet, Agboville est à cheval sur le socle et le domaine sédimentaire dont la limite n'est tranchée de façon verticale.

$\mathrm{Au}$ regard des concordances des résultats dans la majorité des cas, on peut affirmer que l'analyse des fréquences de conductivité électrique permet de déterminer des familles d'eaux pouvant exister dans un aquifère tandis que le diagramme de Piper permet non seulement de déterminer mais de les identifier. Ces deux approches méthodologiques semblent complémentaires à un certain degré et permettent la caractérisation des eaux souterraines.

\section{Conclusion}

L'analyse de la distribution des fréquences de conductivité électrique a fait ressortir diverses familles d'eau. Les zones d'Agboville et de l'ex-région du N'zi-Comoé ont présenté des courbes unimodales symbolisant chacune la présence d'une seule famille d'eau. Par ailleurs, les régions de Tiébissou-Didiévi, Bondoukou et Tiassalé, ont présenté des courbes bimodales symbolisant la présence de deux familles d'eau. La région de Guiglo-Duekoué a montré quatre (4) familles d'eaux régnant au sein de ses aquifères. La projection des échantillons d'eau sur le diagramme de Piper a permis de mettre en évidence différentes familles d'eau présentes dans les régions de Tiébissou-Didievi et d'Agbovile. Au niveau de la région de Tiébissou-Didievi, il est mis en évidence trois familles d'eau qui sont les eaux bicarbonatées, calciques et magnésiennes $(65,38 \%)$, les eaux bicarbonatées sodi-potassiques $(30,77 \%)$ et les eaux chlorurées et sulfatées, calciques et 
magnésiennes (3,85\%). Au niveau d'Agboville, il est mis en évidence quatre familles d'eau qui sont les eaux bicarbonatées, calciques et magnésiennes $(61,90 \%)$, les eaux chlorurées, sodiques et potassiques $(21,43 \%)$, les eaux bicarbonatées, sodiques et potassiques $(9,52 \%)$ et les eaux chlorurées et sulfatées calciques et magnésiennes $(7,14 \%)$. Une comparaison des différents résultats entre les courbes de fréquence de conductivité électrique et les faciès chimiques déterminés à partir du diagramme de Piper a été effectuée. Il ressort de cette comparaison une concordance des résultats au niveau de quatre échantillons (Bondoukou, Tiassalé, ex-N'ziComoé et Tiébissou-Didievi) et une non concordance au niveau de deux échantillons (Guiglo-Duekoué et d'Agboville). Au regard des concordances des résultats dans la majorité des cas, on peut affirmer que l'analyse des fréquences de conductivité électrique permet en général de confirmer les différents faciès hydrogéochimiques en milieux de socle cristallin et cristallophyllien.

\section{RÉFÉRENCES}

Brou YT. 2005. Climat, mutations socioéconomiques et paysages en Côte d'Ivoire. Mémoire de synthèse des activités scientifiques présenté en vue de l'obtention de l'Habilitation à Diriger des Recherches, Université des Sciences et Techniques de Lille, France, p.212.

Fournier M. 2006. Identification du transport et de la vulnérabilité du karst de la craie. Application de nouveaux outils statistiques d'analyse des données au système de Hannetot (Seine-Marine, France). Thèse de Doctorat d'Hydrogéologie karstique à l'Université de Rouen, p. 228.

Ahoussi KE, YouanTa M, Loko S, Adja MG, Lasm T, Jourda JP. 2012. Étude hydrogéochimique des eaux des aquifères de fractures du socle paléoprotérozoïque du Nord-Est de la Côte d'Ivoire: cas de la région de Bondoukou. Afrique Science, Revue
Internationale des Sciences et Technologie, 8(3): 51-68.

Ahoussi KE, Oga YMS, Koffi YB, Kouassi AM, Soro N, Biemi J. 2011. Caractérisation hydrogéochimique et microbiologique des ressources en eau $\mathrm{du}$ site d'un Centre d'Enfouissement Technique (CET) de Côte d'Ivoire : cas du CET de Kossihouen dans le District d'Abidjan (Côte d'Ivoire). International Journal of Biological and Chemical Sciences, 5(5): 2114-2132. DOI : http://dx.doi.org/10.4314 /ijbcs.v5i5.32.

Ahoussi KE, Koffi YB, Kouassi AM, Soro G, Soro N, Biemi J. 2012a. Caractérisation physico-chimique et bactériologique des ressources en eau des localités situées aux abords de la lagune Ébrié dans la commune de Marcory (District d'Abidjan, Côte Ivoire): cas du village d'Abia Koumassi. European Journal of Scientific Research, 89(3): 359-383.

Ahoussi KE, Koffi YB, Kouassi AM, Soro G, Soro N, Biemi J. 2012b. Étude des caractéristiques chimiques et microbiologiques des ressources en eau du bassin versant du N'zi : cas de la commune de N'zianouan (Sud de la Côte d'Ivoire). International Journal of Biological and Chemical Sciences, 6(4): 1854-1873. DOI : http://dx.doi.org/ 10.4314/ijbcs.v6i4.40.

Alayat H, Lamouroux C. 2007. Caractérisation physico-chimique des eaux thermo-minérales des monts de la Cheffia (extrême Nord-Est algérien). $L a$ Presse Thermale et Climatique, 144 : 191-199.

Jourda JPR, Djagoua EV, Kouamé KF, Saley MB, Gronayes C, Achy JJ, Biemi J, Razack M. 2006a. Identification et cartographie des unités lithologiques et des accidents structuraux majeurs du département de Korhogo (Nord de la Côte d'Ivoire) : apport de l'imagerie ETM+ de Landsat. Télédétection, 6(2): 123-142. 
Jourda JPR, Saley MB, Djagoua EV, Kouamé KF, Biemi J, Razack M. 2006b. Utilisation des données ETM+ de Landsat et d'un SIG pour l'évaluation du potentiel en eau souterraine dans le milieu fissuré précambrien de la région de Korhogo (Nord de la Côte d'Ivoire): approche par analyse multicritère et test de validation. Télédétection, 5(4): 339357.

Koita M, Jourde H, Ruelland D, Koffi K, Pistre S, Savané I. 2010. Cartographie des accidents régionaux et identification de leur rôle dans l'hydrodynamique souterraine en zone de socle. Cas de la région de Dimbokro-Bongouanou (Côte d'Ivoire). Journal des Sciences Hydrologiques, 55(5): 805-820.

Kouassi AM, Yao KA, Ahoussi KE, Seki LC, Yao A, Kouassi KI, Biemi J. 2010. Apport des méthodes statistiques et hydrochimiques à la caractérisation des eaux des aquifères fissurés de la région du N'zi-Comoé (Centre-Est de la Côte d'Ivoire). International Journal of Biological and Chemical Sciences, 4(5): 1816-1838.

Kouamelan AN. 1996. Géochronologie et géochimie des formations archéennes et protérozoïque de la dorsale de Man en Côte d'Ivoire - Implications pour la transition Archéen-Protérozoïque ». Thèse de Doctorat Es Sciences Naturelles, Université Rennes I, Mémoires, Géosciences Rennes, $n^{\circ} 73$, p.289.

Kouassi AM, Okaingni JC, Baka D, Lasm T, Kouamé KF, Biémi J. 2012a. Application des méthodes statistiques et géostatistiques à l'étude de la conductivité électrique des eaux souterraines de la région du N'zi-Comoé (Centre-Est de la Côte d'Ivoire). International Journal of Biological and Chemical Sciences, 6(2): 897-912. DOI : http://dx.doi.org/10.4314/ijbcs.v6i2.31.

Kouassi AM, Ahoussi KE, Koffi YB, Aké AY, Biémi J. 2012b. Caractérisation hydrogéochimique des eaux des aquifères fissurés de la zone GuigloDuekoué (Ouest de la Côte d'Ivoire). International Journal of Biological and Chemical Sciences, 6(1): 504-518. DOI : http://dx.doi.org/10.4314/ijbcs.v6i1.45.

Kouassi AM, Kouakou KE, Kadio KKR, Biemi J. 2014. Conception de modèles statistiques à variables hydrogéologiques pour la prévision de la profondeur limite d'arrêt de forage d'eau en milieux de socle en Côte d'Ivoire. International Journal of Innovation and Scientific Research, 11(1): 181-197.

Kouassi AM, Mamadou A, Ahoussi KE, Biemi J. 2013. Simulation de la conductivité électrique des eaux souterraines en relation avec leurs propriétés géologiques: cas de la Côte d'Ivoire. Revue Ivoirienne des Sciences et Technologie, 21\&22: 138-166.

Kouzana L, Ben Mammou A, Gaaloul N. 2007. Intrusion marine et salinisation des eaux d'une nappe phréatique côtière (Korba, Cap-Bon, Tunisie). Geo-EcoTrop., 31: 57-70.

Lasm T, Kouamé K.F., Soro N, Jourda JPR, Biemi J. 2004. Analyse géostatistique de la fracturation extraite de l'imagerie spatiale aéroportée et satellitaire. Application à la région de Man-Danané (Ouest de la Côte d'Ivoire). Revue Ivoirienne des Sciences et Technologie, 5: $135-154$.

Lasm T, Youan-ta M, Jourda JPR, Kouamé KF. 2008. Fracture Networks Analysis in Crystalline Basement: Case of Bondoukou Area (Northeast Côte d'Ivoire). European Journal of Scientific Research, 21(1): 196-208.

Massei N. 2001. Transport de particules en suspension dans l'aquifère crayeux karstique et à l'interface craie/alluvions. Thèse de Doctorat, Université de Rouen, p.189.

Mohammad RAA, Hamed AEN. 2004. Hydrochemical facies of groundwater in the Gaza Strip, Palestine. Journal des Sciences Hydrologiques, 49(3): 359-371. 
N'go YA, Lasm T, Koita M, Savané I. 2010. Extraction par télédétection des réseaux de fractures majeures du socle précambrien de la région de Dimbokro (Centre-Est de la Côte d'Ivoire). Télédétection, 9(1) : 33-42.

Oga MS, Lasm T, Yao KT, Soro N, Saley MB, Kouassi D, Gnamba F. 2009. Caractérisation chimique des eaux des aquifères de Fracture: cas de la région de Tiassalé en Côte d'Ivoire. European Journal of Scientific Research, 31(1): 7287.

Sorokoby VM, Saley MB, Kouamé KF, Djagoua MEV, Bernier M, Affian K, Biemi J. 2010. Utilisation des images Landsat ETM+ et du SIRS pour la cartographie linéamentaire et thématique de Soubré-Meagui (Sud-Ouest de la Côte d'Ivoire): contribution à la gestion des ressources en eau souterraine. Télédétection, 9(3-4): 209-223.

Tabouche N, Achour S. 2004. Etude de la qualité des eaux souterraines de la région orientale du Sahara septentrional algérien. Larhyss Journal, 3: 99-113.

Yao KA, Kouassi AM, Koffi YB, Biemi J. 2010. Caractérisation hydrodynamique et hydrogéochimique des aquifères fissurés de la région de Toumodi (Centre de la Côte d'Ivoire). Journal of Environmental Hydrology, 18(26): 1-15.

Yermani M, Zouari K, Michelot JL, Mamou A, Moumni L. 2003. Approche géochimique du fonctionnement de la nappe profonde de Gafsa Nord (Tunisie centrale). Journal des Sciences Hydrologiques, 48(1): 95-108.

Youanta M, Lasm T, Jourda JP, Kouamé KF, Razack M. 2008. Cartographie des accidents géologiques par imagerie satellitaire Landsat-7 ETM+ et analyse des réseaux de fractures du socle précambrien de la région de Bondoukou (Nord-Est de la Côte d'Ivoire). Télédétection, 8(2) : 119-135. 